Revue d'histoire de l'Amérique française

REVUE D.HISTOIRE DE L'AMÉRIQUE FRANÇAISE

\title{
Papineau et le péril irlandais - 1848
}

\section{Lionel Groulx}

Volume 4, numéro 4, mars 1951

URI : https://id.erudit.org/iderudit/801667ar

DOI : https://doi.org/10.7202/801667ar

Aller au sommaire du numéro

Éditeur(s)

Institut d'histoire de l'Amérique française

ISSN

0035-2357 (imprimé)

1492-1383 (numérique)

Découvrir la revue

Citer cet article

Groulx, L. (1951). Papineau et le péril irlandais - 1848. Revue d'histoire de l'Amérique française, 4(4), 512-520. https://doi.org/10.7202/801667ar d'utilisation que vous pouvez consulter en ligne.

https://apropos.erudit.org/fr/usagers/politique-dutilisation/ 


\section{PAPINEAU ET LE PERIL IRLANDAIS}

\section{8}

Peu de périodes auront été plus fécondes en agitation au Canada que celle de 1848. Gouvernée depuis cinq ans contre ses vœux, la province souffre grandement de son état politique. Les événements d'Europe y ont aussi apporté leur part d'excitation. Les courants révolutionnaires, c'est bien connu, se propagent à travers le monde, à la façon de secousses sismiques. La Monarchie de juillet vient de s'écrouler. Le vieux continent en est ébranlé. L'insurrection éclate à Vienne, à Berlin; la Suisse subit de graves chocs intérieurs; le Pape s'enfuit de Rome; l'Italie, la Hongrie, l'Espagne passent à l'état de volcans.

Que la fièvre révolutionnaire gagne le Royaume-Uni et ne sera-ce point miracle que les Canadas y puissent échapper? La fièvre traverse la Manche. Précisément, en 1848, le "Chartisme" (People's Charter, ou Charter of our rights), mouvement d'émancipation politique des classes laborieuses, tente une de ces vastes manifestations qui, depuis dix ans, inquiètent au plus haut point le public anglais. Les Chartistes prônent le suffrage universel pour hommes, le vote secret, l'abolition du cens d'éligibilité, l'égalité des circonscriptions électorales, des élections annuelles'. L'un des chefs du mouvement, le radical Feargus O'Connor, en relations avec Marx et Engels, projette une énorme assemblée de 200,000 partisans à Kennington Common, qui iraient porter au Parlement une pétition appuyée de 2,000,000 de signatures. Des nouvelles encore plus alarmantes viennent de l'île voisine où le volcan toujours mal assoupi se remet à

1. John Richard Green, A History of the English People (5 vol., New York, Philadelphia, Chicago, s.d.), V: 67-69. - Elie Halévy, Histoire du Peuple anglais au XIXe siècle (7 vol., Paris, 1913-19 ), III: 255-334. - Charlotte M. Waters, $A$ Short Economic History of England (2 vol., Oxford University Press, 1925), II: $564-572$. 
gronder. En 1847 y a pris naissance le parti de la "Jeune Irlande", en réaction contre le modérantisme d'O'Connell vieillissant. Elevés dans les souvenirs de la Grèce républicaine, nourris des Girondins de Lamartine, bréviaire de tous les révolutionnaires de l'époque, quelques jeunes Irlandais n'en tiennent plus que pour le recours aux méthodes de violence. Issus presque tous de familles riches, doués de remarquables talents, leur mouvement prend un essor rapide, surtout lorsque à la tête vient se placer William Smith O'Brien, grand propriétaire et grand seigneur, frère de lord Inchiquin, et rattaché, par ses ancêtres, au passé légendaire de sa race. D'ailleurs O'Connell est mort le 15 mai 1847, laissant le champ libre à cette jeunesse impatiente. Devant l'insurrection grondante, force sera au parlement britannique de recourir aux grands moyens: le vote d'une loi d'urgence et la suspension de l'habeas corpus'.

Qui serait supris d'une large répercussion de ces événements au Canada? Un malaise, et peut-être une frayeur qu'on peut appeler proprement le péril irlandais, tient en haleine, en 1848, les autorités politiques. A vrai dire, ce péril hante depuis quelques années l'esprit des gouvernants. En 1845 sir Charles Metcalfe attire l'attention du gouvernement métropolitain sur le danger d'une trop considérable immigration d'Irlandais catholiques. "Les Irlandais catholiques romains", écrit-il à Lord Stanley, "composent la grande majorité des émigrants du Royaume-Uni, et par conséquent, suivant toute apparence, le parti des mécontents dans la province augmentera de façon beaucoup plus rapide que celui de la population loyale, ce qui pourra par la suite produire des effets désastreux". Sir Charles émet donc le souhait que l'on oriente plus sagement l'immigration des Iles britanniques: les Irlandais catholiques seraient dirigés de préférence vers les colonies de tout repos; aux Canadas, pays troubles, on réserverait les éléments irréprochables, la fine fleur du loyalisme, c'est-à-dire les colons anglais et les Irlandais protestants, ces derniers même de préférence aux Ecossais ${ }^{3}$. Soit négligence ou autre cause,

2. Voir, pour l'histoire de la "Jeune Irlande", Mac Carthy, Histoire contemporaine d'Angleterre depuis l'avènement de la reine Victoria jusqu'aux élections générales de 1880 (1837-1880) (5 vol., Paris, 1885), II: 15-32. - I. Nemours Godré, Daniel O'Connell, sa vie, son ceuvre (Paris, 1900), 383-387.

3. A. Gérin-Lajoie, Dix Ans au Canada de 1840 d̀ 1850 - Histoire de l'établissement du gouvernement responsable (Québec, 1888), 298-299. 
les avertissements de Sir Charles Metcalfe ne trouvent pas oreilles ouvertes. Si bien qu'en 1848, Canadiens français et Irlandais forment le plus gros de la population de la province "... nearly half of the population of this Colony are French - nearly half of the remainder Irish-", écrivait Elgin 4 . Dans une note secrète, le gouverneur fait observer à Grey que cinq Irlandais, MM. Sullivan, Hincks, Blake, Drummond, Baldwin, font ou feront partie du Conseil exécutif de $1848^{5}$. C'est alors au tour de lord Elgin de s'émouvoir. Et les inquiétudes du noble lord se fortifient de tout ce que lui apportent les nouvelles d'Europe. Entre l'Ile verte et la Grande-Bretagne, vient de se ranimer le duel séculaire. Les événements d'outre-mer agissent au Canada, sur les masses irlandaises, à la façon de violents corrosifs. "Jeune Irlande" et groupe plus pacifique du "Rappel" comptent d'enthousiastes partisans parmi ces parias qui, en leur léger bagage, ont emporté, de ce côté-ci de l'océan, toute autre chose qu'un amour immodéré de l'Angleterre. Au printemps de 1848, l'on croit discerner d'assez graves symptômes: à Québec et à Montréal, tenues d'assemblées qu'on dit monstres, votes de résolutions frondeuses, fondations de club ou de ligue, fondation même d'un journal, prestation de serments secrets, enfin bruit d'armements. A Montréal, les autorités militaires, très énervées, prêtent une docile oreille aux rumeurs les plus alarmistes. Esprits apeurés voient volontiers avec verres grossissants; on parle donc d'émissaires qui seraient passés du Canada à Boston, pour organiser, en ce dernier endroit, des sociétés ou clubs irlandais, cueillir des fonds, soudoyer des bandes d'invasion. Déjà 5,000 hommes se tiendraient prêts à franchir la frontière; 15,000 autres seraient en voie de s'enrôler à New-York ${ }^{6}$. On va jusqu'à parler d'une attaque probable sur l'Hôtel du gouvernement canadien et sur les magasins militaires. Pendant de longs mois la correspondance de lord Elgin s'emplit de ces appréhensions qu'elle colporte à Londres.

Nouvel arrivé au pays, Elgin juge alors hommes et choses un peu confusément. Plus encore que des rêveurs d'insurrection, il fait

4. Elgin à Grey, 26 avril 1848, The Elgin-Grey Papers 1846-1852, Edited with notes and appendices by Sir Arthur G. Doughty (4 vol., Ottawa, 1937), I: 145.

5. Ibid., I: 161-162. I: 281 .

6. Ibid., Elgin à Grey, 24 aout 1848, I: 226; lettre à Elgin, Boston, 26 déc. 1848, 
le compte de leurs alliés possibles. Et il appréhende de troublantes complications. A cette époque, un élément ne rassure qu'à demi le gouverneur: l'élément canadien-français. Il le voit toujours sourdement irrité par le coup de force de 1841, tendant une oreille complaisante aux moindres bruits de révolution qu'apportent les courriers de France. L'attitude des Canadiens français serait celle d'une "neutralité armée". Ne sont-ils pas les victimes du "British égö̈sme"? L'Union des Canadas n'a-t-elle pas été votée sans leur consentement et pour les soumettre à la population britannique? Rengaines que leurs journaux, leurs chefs, et surtout Papineau, ne cessent de leur rabâcher ${ }^{7}$. Plus que tout le reste, en effet, sur cette masse apparemment indécise, Elgin redoute l'influence de Louis-Joseph Papineau. L'ancien chef était rentré au pays depuis 1845 . L'homme avait alors soixante ans, qu'il portait allégrement. Sur quel plan allait-il recommencer sa vie? Ce parlementaire de carrière pourrait-il s'empêcher de retourner à la vie politique? Et s'il réapparaissait sur la scène, sous quelles couleurs, sous quelle étiquette, lui plairait-il de se ranger? La question passionne ses anciens amis et ennemis qui savent toujours considérable le prestige du grand orateur sur la foule. Luimême, dans les premiers mois de son retour, semble prendre plaisir à tenir en suspens la curiosité publique. Il multiplie discours et attitudes énigmatiques, distribue à tous les partis des gages et des blâmes, qu'entre les uns et les autres il balance habilement. Bientôt néanmoins il devient évident que si l'exil a peu appris au grand homme, il n'a pas davantage oublié. Mal ou travers, comme l'on sait, qui n'a rien de proprement bourbonnien, mais qui serait le lot de tous les proscrits longtemps retenus hors de leur pays. Le manifeste de Papineau, à la veille des élections de 1848, déchire le voile. Elgin n'y voit qu'“a pretty frank declaration of Republicanism... the writer's hatred of the British Gov ${ }^{t}$ - his distrust of Responsible Gov'..."8 Un seul sentiment a changé chez Papineau, mais pour se développer jusqu'à l'hypertrophie: sa vieille aversion du régime colonial britannique, aversion où il ne se prive pas d'envelopper l'Angleterre elle-même. "Leur Acte d'Union, écrira-t-il, par exemple, des gouvernants métropolitains, "est la plus grossière des iniquités

7. Ibid., Elgin à Grey, 16 aout 1848, I: 224.

8. Ibid., I: 78. 
commises durant toute la période de leur histoire coloniale. Le nom de colon britannique est celui des esclaves politiques les plus avilis..." En ce régime, Papineau voit la source unique de tous les maux dont souffre le pays et de même l'insurmontable obstacle à toute réforme et à tout progrès. Il en viendra à écrire: "La défection est si générale que je ne suis dans la vie publique qu'avec une répugnance et un dégout de tous les instants, pendant que je suis la voix qui crie dans le désert, sans le plus léger espoir d'obtenir justice ni réforme, tant que nous serons colonies ${ }^{10}$ ". De là à souhaiter la politique du pire, il n'y avait qu'un pas. Ce pas, l'irréductible mécontent aura bientôt fait de le franchir. Dans le malaise suprême, il saluera le suprême moyen de libérer les Canadas de l'abjection coloniale: "Plus vite s'useront gouverneurs et ministres", applaudira-t-il, "plus vite s'usera la domination anglaise, seule cure suffisante à nos maux.."

Que ce langage et ces poses de prophète désabusé fussent de nature à semer l'émoi dans le monde officiel et jusque parmi les lieutenants de l'ancien chef, inutile de l'affirmer. Gagnés à d'autres méthodes de gouvernement, quelques-uns s'affligent de voir compromise l'œuvre de pacification en voie de s'accomplir entre les races. De ce retour aux anciennes et stériles agitations, qu'attendre sinon l'ajournement de la politique constructive enfin possible depuis la conquête du gouvernement responsable? Pour quelques autres, c'est le régime même de l'Union qui leur paraît mis en question, en train de chanceler. Une question se pose donc, et non sans quelque anxiété, à l'esprit d'un grand nombre et en particulier d'Elgin: quelles seront bien sur les masses, sur l'opinion, les prises du terrible agitateur? Qu'à l'ensemble de ses compatriotes ou qu'à tous les libéraux désenchantés, il parvienne à rallier l'élément irlandais, quel avenir troublé ne pas présager? Or, justement, en ce printemps de 1848, divers indices font craindre la possibilité d'une alliance entre les deux groupes de la population ${ }^{12}$. Papineau laisse inscrire son

9. L.-J. Papineau au Dr O'Callaghan, 28 octobre 1846, Archives de l'auteur. (Il s'agit de copies transcrites, il y a quelques années par l'auteur, sur les originaux au Manoir de Montebello).

10. L.-J. Papineau au Dr O'Callaghan, ler février 1850, Archives de l'auteur.

11. L.-J. Papineau au Dr O'Callaghan, ler février 1850, Archives de l'auteur.

12. Elgin à Grey, 26 avril 1848, The Elgin-Grey Papers 1846-1852, Edited with notes and appendices by Sir Arthur G. Doughty (4 vol., Ottawa, 1937), I: 144-145. 
nom comme vice-président de la Ligue irlandaise. Il accepte de participer aux assemblées de la Ligue tenues à Québec et à Montréal. Ses sympathies, Papineau les accorde d'autant plus volontiers à l'antique victime de la politique anglaise, qu'avec son imagination grossissante, il en discerne sans peine, en sa petite patrie, une réplique infortunée. Dans sa correspondance avec son ancien ami, O'Callaghan, il sera question du "Canada immolé comme l'Irlande, au système de corruption universelle par lequel l'Angleterre souffre chez elle, et veut, dans toutes ses dépendances, l'exploitation des masses au profit du petit nombre et cela avec plus d'avidité et de suite, qu'elle n'est pratiquée aujourd'hui par aucun autre des Gouvernements de la Chrétientél's". Il écrit encore au même: "D'ici à peu d'années, nous serions par rapport au Haut-Canada, dans la même situation qu'est l'Irlande par rapport à l'Angleterre ${ }^{14 "}$. Prompt, trop prompt à s'alarmer, Elgin qui suit de très près les agissements de Papineau, perd, lui aussi, la mesure. Il croit marcher sur un sol miné. Il appréhende une crise tragique où le pays ne devra qu'à son heureuse chance d'échapper ${ }^{15}$.

Nous avons dit le peu de cas qu'il fait pour le moment de la loyauté canadienne-française. Combien plus suspects toutefois lui apparaissent les milieux tories, où Lord Elgin ne rencontre pas seulement du mécontentement, mais la plus coléreuse aigreur contre la métropole et sa récente évolution économique et commerciale: thoroughly disgusted and lukewarm in their allegiance, if not dessaffected. Stupéfiante évolution de ces anciens janissaires de l'orthodoxie loyaliste qui en viennent à souhaiter ouvertement la rupture du lien colonial! Le gouverneur en est à ce point bouleversé qu'il estime bonheur sans pareil, pour les Canadas, de n'être plus sous les rênes d'une administration tory ${ }^{16}$.

Le malheur veut que la cause irlandaise ne se recrute point de partisans qu'à l'intérieur de la province. Ce n'est pas vainement

13. L.-J. Papineau au Dr O'Callaghan, 27 mars 1848, Archives de l'auteur.

14. L.-J. Papineau au Dr O'Callaghan, 22 février 1848, Archives de l'auteur.

15. Elgin à Grey, 4 mai 1848, The Elgin-Grey Papers 1846-1852, Edited with notes and appendices by Sir Arthur G. Doughty (4 vol., Ottawa, 1937), I: 148-150. $263-265$.

16. Ibid., Elgin a Grey, $18 \mathrm{mai}, 16$ aodt et 30 novembre 1848, I: 223-225, 
qu'intrigues se sont nouées par-dessus les frontières. Des émissaires dépêchés par les associations irlandaises de New-York opèrent, à ce qu'il semble bien, dans les deux Canadas ${ }^{17}$. Pour porter secours à l'insurrection de la "Jeune Irlande", projetée pour les mois prochains, un nommé O'Connor, éditeur d'un journal de New-York, serait même venu, à l'été de 1848, préparer une diversion au Canada. Cinquante mille Irlandais, postés d'après lui près de la frontière américaine, n'attendraient qu'un signe pour se mettre en marche vers le nord ${ }^{18}$. Et puisque l'on n'en est pas à une rumeur près, la nouvelle se répand de l'arrivée prochaine de convois d'armes et de munitions expédiés par les Américains. Ce qui est sûr, c'est que l'Etat voisin de New-York est en ébullition. Clubs à sympathies irlandaises s'y multiplient. Des manifestations considérables s'organisent où l'on prélève des fonds pour la libération de l'Irlande. Mieux encore: les politiciens américains courtisent ouvertement cette effervescence. Les candidats à l'élection présidentielle s'essaient de leur mieux à capter le vote irlandais. L'un d'eux, plus audacieux que les autres, se déclare favorable à l'annexion du Canada aux Etats-Unis. A Washington et à New-York, remués par de tels propos et de tels agissements, le chargé d'affaires et le consul anglais prennent peur tout de bon ${ }^{19}$. Un vent d'orgueil souffle d'ailleurs sur la nation américaine. Le drapeau étoilé vient d'entrer dans Mexico: entrée triomphale qui aura pour suite la conquête du Texas, du NouveauMexique et de la Californie. Grisée de ses succès et de ses accroissements de territoire, la jeune nation s'abandonne volontiers à des rêves de gloire militaire. La guerre finie et une partie de l'armée licenciée, des bandes de soldats désœuvrés errent à travers les Etats et remontent vers le nord. Ne serait-ce point-là, se demande-t-on de ce côté-ci de la frontière, les envahisseurs tout désignés que la première occasion fera apparaître aux portes du pays ?20 $\mathrm{Au}$ début de l'automne de 1848, l'émoi est tel que les autorités se déterminent avec une hâte fébrile à des mesures défensives. A la moindre alerte, deux régiments de volontaires seront levés, l'un anglais, l'autre

17. Ibid., Elgin à Grey, 16 novembre 1848, I: 256-257.

18. Ibid., Elgin à Grey, 15 juillet 1848, I: 209-210.

19. Ibid., I: 224.

20. Ibid., Elgin à Grey, 16 et 24 aout 1848, I: 223-224; 226. 
français. Ces deux seront chargés de la garde de Montréal. Pendant ce temps-là, relevée de sa faction, la garnison de la ville se portera promptement à la frontière pour y écraser les premières bandes.

L'on en était là, dans l'attente des pires événements, lorsque tout à coup quelques nouvelles d'Europe vinrent dissiper le cauchemar. L'assemblée de 200,000 partisans projetée par Feargus O'Connor à Kennington Common, ne réussissait à grouper qu'à peine 25,000 personnes. La pétition qui devait porter 2,000,000 de signatures, au Parlement, en portait 2,00021. Le mouvement de la "Jeune Irlande" échouait misérablement, comme toutes ces tentatives de jeunes hommes où il entre plus d'enthousiasmes et de paroles que de prévoyance et d'action. A la suite d'une ou deux escarmouches assez pitoyables, tous les chefs, y compris O'Brien, étaient capturés, jetés en prison, déportés, ou prenaient d'eux-mêmes le chemin de l'exil. Et c'est ainsi, pour le noter en passant, qu'arrivait un jour au Canada, un jeune Irlandais à qui les événements réservaient un certain rôle, Thomas d'Arcy McGee.

$\mathrm{Au}$ Canada, les partisans d'O'Brien auraient-ils meilleur succès? Désaccords ni tiraillements ne leur avaient manqué. Les partisans du simple "Rappel" irlandais répugnaient à se commettre avec les extrémistes de la république irlandaise. Ces derniers se recrutaient, par malheur, dans la catégorie des plus pauvres immigrants. De ces parias, leurs compatriotes plus à l'aise s'éloignaient comme de pestiférés. Une vaste assemblée ("monster meeting") organisée pour le 6 juillet 1848 à Montréal, par O'Connor, sous les auspices de l'Union républicaine irlandaise de New-York, aboutissait à un complet échec. Personne de notable parmi les partisans du Rappel irlandais ne s'y était montré. Un violent orage électrique avait dispersé les quelque cents auditeurs ${ }^{22}$. Quelques mois plus tard, Elgin pouvait écrire à Lord Grey: "Nous avons eu, nous aussi, notre agitation irlandaise. Elle a seulement fourni l'occasion au groupe des respectables partisans du Rappel de protester contre l'introduction des "Irish Politics" au Canada" 23 . Sans argent et sans autres chefs

21. John Richard Green, A History of the English People (5 vol., New York, Philadelphie, Chicago, s.d.), V: 69.

22. The Elgin-Grey Papers 1846-1852, Edited with notes and appendices by Sir Arthur G. Doughty (4 vol., Ottawa, 1937), I: 205, 210.

23. Ibid., I: 265. 
que des aventuriers, le mouvement irlandais au Canada était donc voué, lui aussi, au lamentable avortement. Après l'échec d'O'Brien, ce fut un jeu, pour les autorités canadiennes, que de tourner contre ce mouvement la presse des deux langues. Papineau lui-même, les premiers élans passés, se prêtait plus qu'il ne se donnait et se prêtait même avec des reprises et des méfiances. Vivement dénoncés, les émissaires américains n'eurent plus que le choix de reprendre le chemin de leur pays, cependant qu'à la frontière, les rêveurs d'invasion, menacés du traitement des brigands, jugeaient prudent de se tenir cois.

Ainsi prit fin cette alerte qui, pendant six mois, avait tenu en haleine Elgin et son entourage. Elle prenait fin assez brusquement. Et l'on peut même conjecturer qu'à l'imitation de certain personnage de la fable, les gouvernants, une fois le péril passé, s'amusèrent quelque peu de leur frayeur.

Lionel Groulx, ptre

N.B. Avec cette livraison de mars de la Revue, prend fin votre abonnement pour l'année 1950-1951. Vous vous ferez, sans doute, un devoir de le renouveler le plus tôt possible. 